ARTí́CULO CIENTÍFICO

\title{
CARACTERÍSTICAS TAXONÓMICAS DE PASTOS BRACHIARIA UTILIZADOS EN COSTA RICA ${ }^{1}$
}

Luis Villalobos-Villalobos ${ }^{2}$, Mayra Montiel-Longhi ${ }^{3}$

\section{RESUMEN}

Se evaluó las características taxonómicas de 13 cultivares de pasto del género Brachiaria de uso común en Costa Rica; tres cultivares de B. decumbens (Basilisk CIAT 606, Peludo y Basilisk) y diez de B. brizantha (Piatá, MG-5 Victoria, Diamantes 1-CIAT 6780, Basilisk, Mulato-CIAT 36061, Mulato 2-CIAT 36087, MG-5 Toledo, Marandú, Toledo-CIAT 26110 y Xaraes-Toledo). Se cosecharon plantas enteras en estado vegetativo (hojas y culmos) y en estado de floración (inflorescencias y espiguillas) de cada cultivar. El largo y ancho de las hojas fueron entre 20-68 cm y 1 a 2,2 cm, respectivamente. El largo de los culmos fue de 0,23 a 2,5 $\mathrm{m}$ y el largo de los entrenudos entre 12 a $34 \mathrm{~cm}$. La cantidad de racimos por inflorescencia fue entre 2 a 5 y cada uno con un largo entre 7 a $23 \mathrm{~cm}$. El largo total de las inflorescencias y de sus vainas fue 23 a $55 \mathrm{~cm}$ y 8,9 a $35 \mathrm{~cm}$, respectivamente. El largo de las espiguillas fue $5 \mathrm{a} 8 \mathrm{~cm}$. La información recolectada en este estudio pretende ser una herramienta de campo para la identificación de cultivares de Brachiaria uso común en Costa Rica.

Palabras clave: morfología, taxonomía, forrajes, pasturas tropicales, Brachiaria

\section{ABSTRACT}

Taxonomic traits for Brachiaria grasses used in Costa Rica. Taxonomic traits of 13 grass cultivars of the genus Brachiaria were evaluated; 3 cultivars from the species $B$. decumbens (Basilisk CIAT 606, Peludo y Basilisk) and ten from B. brizantha (Piatá, MG5 Victoria, Diamantes 1-CIAT 6780, Basilisk, Mulato-CIAT 36061, Mulato 2-CIAT 36087, MG-5 Toledo, Marandú, Toledo-CIAT 26110 y Xaraes-Toledo). Whole plants were

\footnotetext{
${ }^{1}$ Inscrito en Vicerrectoría de Investigación. Universidad de Costa Rica. Proyecto 739-A9-182

${ }^{2}$ Universidad de Costa Rica. Escuela de Zootecnia y Centro de Investigación en Nutrición Animal. Autor para correspondencia: Luis.villalobosvillalobos@ucr.ac.cr

${ }^{3}$ Universidad de Costa Rica. Escuela de Zootecnia

Recibido: 23 de marzo 2015

Aceptado: 27 de abril 2015
} 
harvested in a vegetative stage (leaves and culms) and in a flowering stage (flowers and spikelets)for each cultivar. The length and width of leaves were 20 to $68 \mathrm{~cm}$ and 1 to 2.2 $\mathrm{cm}$, respectively. The length of the culms was 0.23 to $2.5 \mathrm{~cm}$ and the length of single internodes was 12 to $34 \mathrm{~cm}$. The inflorescences contained 2 to 5 racemes each with a length of 7 to $23 \mathrm{~cm}$. The length of inflorescences and its sheaths was 23 to $55 \mathrm{~cm}$ and 7 to $23 \mathrm{~cm}$, respectively. The length of spikelet's was 5 to $8 \mathrm{~cm}$. Data collected in this study is intended to provide a field tool for identification of Brachiaria grass cultivars used in Costa Rica.

Key words: morphology, taxonomy, forages, tropical pastures, Brachiaria

\section{INTRODUCCIÓN}

Las especies del género Brachiaria son originarias de las regiones tropicales de África en donde crecen normalmente de forma natural en sabanas abiertas o en compañía de especies arbustivas. Pertenecen a la división Magnoliophyta; clase Magnoliopsida, subclase Commelinidae; orden Poales; familia Poaceae; subfamilia Panicoideae; tribu Paniceae (Olivera et al., 2006). Dicho género contiene alrededor de 100 especies que crecen en regiones tropicales y subtropicales en los hemisferios Oriental y Occidental (Zuleta y Cardozo, 2002). El género Brachiaria reúne las especies más utilizadas como forrajeras en América tropical tales como B. brizantha cv. Marandú, Toledo y La Libertad; B. decumbens cv. Basilisk; B. humidicola y B. ruziziensis cv. Kennedy (Argel, 2006; Zuleta et al., 2002).

Los pastos Brachiaria muestran gran aceptación por parte de los ganaderos debido a su adaptación a diversas condiciones edafo climáticas. Bajo condiciones limitantes en el suelo como acidez y baja fertilidad, los pastos del género Brachiaria muestran un eficiente crecimiento y persistencia, así como altas producciones de biomasa de buena calidad y un alto grado de aceptación por los animales (Olivera y Del Pozo, 2006). Hernández y Herrera (2005) encontraron diferentes grados de respuesta de los pastos $B$. decumbens y $B$. brizantha a la aplicación de herbicidas graminicidas, lo cual permite que los procesos de renovación de pasturas sean más efectivos para eliminar la competencia de otras gramíneas invasoras en los potreros, lo cual finalmente limita la productividad de los pastos. WingChing et al. (2008), encontraron una baja 
susceptibilidad al ataque de nemátodos por parte del pasto $B$. decumbens, evidenciado por la alta producción de biomasa y la larga vida útil del cultivo. Dichas características de adaptabilidad del género Brachiaria son fundamentales para soportar las características climatológicas en el trópico como la irregularidad en la distribución de las lluvias, la disminución en precipitación y el aumento en temperatura; que finalmente afectan el comportamiento productivo y reproductivo del ganado bovino (Retana y Rosales 2000).

Recientemente, los pastos Brachiaria han despertado mayor interés como medio para reducir la producción de gases de efecto invernadero, debido a su capacidad inhibitoria de la nitrificación biológica tal como ocurre con B. humidicola, dicho mecanismo permitiría minimizar las pérdidas del nitrógeno aplicado por medio del fertilizante (CIAT, 2010).

En América Latina se cuenta con cerca de 80 millones de hectáreas de pastos Brachiaria, siendo el género que contribuye con más materiales forrajeros; se estima que su utilización como fuente alimenticia, ha ayudado a salir de la pobreza a productores pecuarios del sudeste asiático y América Central en un período de 1 o 2 años (CIAT, 2010; Zuleta et al., 2002). De acuerdo con Carrillo (2012) existen diez empresas nacionales dedicadas a importar semilla de especies forrajeras las cuales reportaron en el año 2012 un ingreso de $386478 \mathrm{~kg}$ de semilla, siendo las principales especies Brachiaria brizantha cv. Diamantes 1 (88210 kg), Mucuna sp. (57370 kg), Panicum maximum cv. Mombaza (48805 kg), Brachiaria brizantha cv. Toledo (36480 kg) y varios cultivares de ryegrass (Lolium sp. $65889 \mathrm{~kg}$ ). El monto de las importaciones de semilla de especies forrajeras para el año 2012 fue de \$3.526.497, del cual las especies del género Brachiaria representan un 60,7\%. Dicho porcentaje aumentó con respecto al año 2010 donde dicho género representó un $40 \%$ del costo de las importaciones de semilla a Costa Rica; siendo Brasil el país de origen del $89,9 \%$ de las importaciones.

En los últimos 10 años, han ingresado a Costa Rica una mayor variedad de cultivares de pastos del género Brachiaria, debido a que se han liberado nuevas accesiones por parte del Centro de Investigaciones en Agricultura Tropical (CIAT) al mercado (Argel, 2006), asimismo, se reporta un leve repunte en la actividad ganadera de carne y leche para el mismo período acompañado del aumento en los precios de las materias primas 
utilizadas para elaborar alimentos balanceados (Carillo, 2012).Los pastos mejorados, incluyendo los del género Brachiaria, permitieron incrementar la productividad en sistemas pecuarios en la década 1990-2000 en la región de Centroamérica y México; particularmente en Costa Rica el impacto se considera mayor con un 55\% de aumento en leche y $18 \%$ en carne (Argel, 2006). Se considera que muchos ganaderos en el país se han visto motivados a renovar pasturas y hacer un uso más eficiente por tratarse de un recurso barato con capacidad de incrementar la productividad en sus fincas (Villalobos et al., 2013).

Muchos de los cultivares que han ingresado al país, se manejan a nivel comercial con nombres comunes genéricos tal como: "Brachiarias", "Brizantha", "Decumbens", "Toledo", "Pasto peludo", "Mulato", entre otros. La utilización de nombres vernáculos es común entre comerciantes, productores y técnicos, muchos de ellos son nombres de uso común en otras latitudes, principalmente del país de origen de la semilla, los cuales pueden diferir de los utilizados en el país importador, lo cual finalmente puede confundir a los consumidores en el momento de compra. La escogencia de los cultivares adecuados para las condiciones ambientales de las fincas se vuelve igualmente compleja debido a la gran cantidad de información técnica disponible luego de intensos procesos de selección y mejoramiento genético en temas como tolerancia a plagas, enfermedades y sequía; así como el efecto directo sobre el rendimiento del ganado bovino (Zuleta et al., 2002; WingChing et al., 2008; Retana y Rosales, 2000).

El uso de herramientas de identificación de pastos permite a los ganaderos realizar compras más certeras. Los pastos mejorados tienen capacidad de mejorar la fertilidad del suelo y mejorar la nutrición humana en zonas marginales al incrementar la producción de leche y/o carne bajo condiciones extremas de sequía y suelos ácidos (CIAT, 2010). Asimismo, la semilla es solo una parte del costo de establecimiento de pasturas y dicho rubro puede ser significativo dependiendo del tipo de cultivo forrajero (Villalobos y WingChing, 2014). A nivel nacional no existen herramientas que permitan identificar los cultivares de pasto del género Brachiaria.

Solo algunas especies de pastos del género Brachiaria muestran características propias, tal como sucede con Brachiaria decumbens y Brachiaria brizantha, el primero se conoce como "pasto peludo" debido a la pubescencia en sus culmos* y su

\footnotetext{
* Al final del artículo se encuentra un glosario de términos vara facilitar la lectura
} 
crecimiento decumbente que le permite formar una cobertura densa sobre el suelo (Cook et al., 2005 y Olivera et al., 2006).

Sin embargo, como se mencionó antes, existen en el mercado varios cultivares de cada una de dichas especies y no se cuenta con información sobre sus características morfológicas que sirvan como parámetros de identificación.

Debido a que no siempre se cuenta con validaciones de especies forrajeras en el país y, que las empresas comercializadoras de semilla brindan información proveniente de otras latitudes, el desarrollo de claves taxonómicas de identificación en campo contribuye a que los productores puedan tener mayor certeza sobre las semillas adquiridas.

El objetivo de la presente investigación fue evaluar características morfológicas que sirvan de herramienta para la identificación taxonómica de los cultivares de pasto del género Brachiaria.

\section{MATERIALES Y MÉTODOS}

El proyecto se realizó en la Estación Experimental Fabio Baudrit Moreno (EEFBM) de la Universidad de Costa Rica (UCR), ubicada en el Distrito San José de Alajuela a 840 msnm, con una precipitación anual promedio de 1763,5 mm distribuidos de mayo a noviembre y temperatura anual promedio de $22^{\circ} \mathrm{C}$, con temperaturas mínima y máxima de 18,2 y $30,2^{\circ} \mathrm{C}$, respectivamente (IMN 2012; EEFBM 2014). De acuerdo con registros de precipitación del Instituto Meteorológico Nacional del año 1996 al 2012, la precipitación anual en la región de Alajuela es de 1763,5 mm y se clasifica como Bosque Húmedo pre montano según las zonas de vida de Holdridge (Janzen, 1991).

\section{Escogencia de semillas, terrenos y establecimiento}

Se establecieron 13 parcelas de $3 \mathrm{~m}$ x $3 \mathrm{~m}$ con pasillos de 1,25 m (Figura 1) y se utilizaron dosis recomendadas para la siembra (6-8 kg.ha-1). Debido a que la cantidad de semilla requerida fue pequeña, la Oficina Nacional de Semillas brindó la mayoría de 
los cultivares por medio de la custodia que maneja el Centro de Investigaciones en Granos y Semillas (CIGRAS) de la UCR.

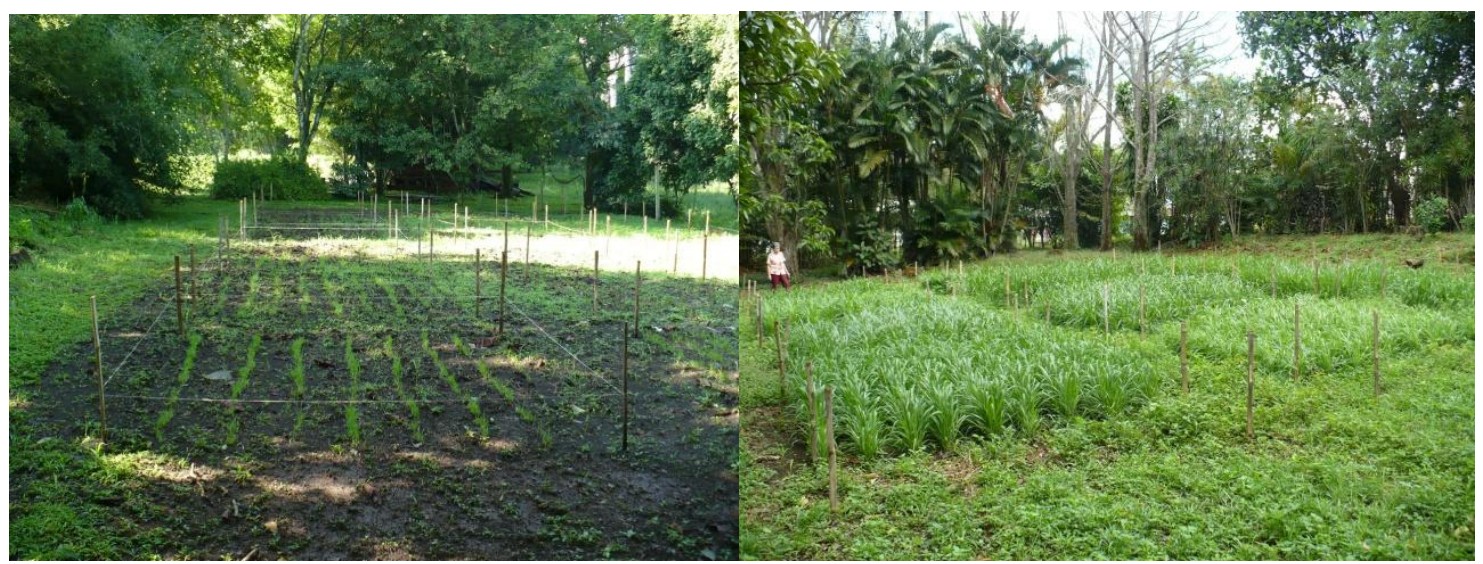

Figura 1. Parcelas para la recolección del material evaluado.

Asimismo se contó con información sobre los indicadores de germinación en cada cultivar (Cuadro 1). Se preparó el terreno por medio de limpieza mecánica y se realizaron surcos cada $8 \mathrm{~cm}$, se depositó la semilla y se cubrió con suelo. En los meses de verano se utilizó riego para el mantenimiento de las parcelas.

Cuadro 1. Cultivares de Brachiaria y sus características al momento de establecimiento.

\begin{tabular}{lcccc}
\hline Parcela & Especie & Variedad & \% germinación & Accesión \\
\hline 1 & Brachiaria decumbens & Basilisk & 65 & CIAT 606 \\
2 & Brachiaria decumbens & Peludo & 76 & \\
3 & Brachiaria decumbens & Basilisk & 67 & \\
4 & Brachiaria brizantha & Piatá & 83 & \\
5 & Brachiaria brizantha & MG-5 Victoria & 22 & CIAT 6780 \\
6 & Brachiaria brizantha & Diamantes 1 & 80 & CIAT \\
7 & Brachiaria brizantha & Basilisk & 72 & 36061 \\
8 & Brachiaria brizantha & Mulato & 69 & CIAT \\
& Brachiaria brizantha & Mulato 2 & 72 & \\
9 & Brachiaria brizantha & MG-5 Toledo & 4087 \\
10 & Brachiaria brizantha & Marandú & 63 & CIAT \\
11 & Brachiaria brizantha & Toledo & 71 & 26110 \\
13 & Brachiaria brizantha & Xaraes-Toledo & 82 & \\
\hline
\end{tabular}




\section{Elaboración de claves taxonómicas}

Se recolectó material vegetativo de las parcelas para cada uno de los cultivares con el fin de elaborar los análisis taxonómicos a partir de las características morfológicas de las hojas y los culmos (Cuadro 2).

Cuadro 2. Características morfológicas de las hojas y el culmo de 13 cultivares de Brachiaria sp.

\begin{tabular}{|c|c|c|c|c|c|}
\hline & \multicolumn{3}{|c|}{ Hoja } & \multicolumn{2}{|c|}{ Culmo } \\
\hline Especie-cultivar & Ancho $(\mathrm{cm})$ & Largo $(\mathrm{cm})$ & $\begin{array}{l}\text { Pubescente } \\
\text { (P) o } \\
\text { Glabra (G) }\end{array}$ & $\begin{array}{c}\text { Largo } \\
\text { entrenudo } \\
\text { (cm) }\end{array}$ & $\begin{array}{l}\text { Largo total } \\
\text { (m) }\end{array}$ \\
\hline B. decumbens-Basilisk & 2,7 & $20-40$ & G (áspera) & $12-20$ & 1,15 \\
\hline B. decumbens-Peludo & $1,8-1,9$ & $37-38$ & G (áspera) & $13-19$ & 0,23 peludo \\
\hline B. decumbens-Basilisk & $1,0-1,8$ & - & G (áspera) & $13-19$ & 0,77 \\
\hline B. brizantha-Piatá & 1,5 & $63-64$ & $P$ & $20,5-21,0$ & - \\
\hline B. brizantha-MG-5 Victoria & $1,5-2,0$ & $61-68,53$ & G (áspera) & $25,5-34,0$ & 1,29 \\
\hline B. brizantha-Diamantes1 & 1,0 & 40 & $P$ & 23 & 1,24 \\
\hline B. brizantha-Basilisk & 1,4 & 42 & $P$ & 23,5 & 1,40 \\
\hline B. brizantha-Mulato & 1,0 & 25 & G & 16,5 & 1,20 \\
\hline B. brizantha-Mulato 2 & $1,0-1,8$ & 30 & G (áspera) & $17,5-19,0$ & 0,96 \\
\hline B. brizantha-MG-5 Toledo & 1,5 & 31 & $\mathrm{G}$ & $13-20$ & 1,16 \\
\hline B. brizantha-Marandú & $1,6-2,0$ & $40-59$ & $\mathrm{G}$ & 16 & 2,50 \\
\hline B. brizantha-Toledo & $1,0-2,0$ & 39 & $\mathrm{G}$ & 24 & 1,30 \\
\hline B. brizantha-Xaraes-Toledo & $2,0-2,2$ & 62 & $G$ & 20 & 1,78 \\
\hline
\end{tabular}

La recolección de inflorescencias se realizó hasta que cada cultivar alcanzó el estado de madurez ( 3 meses en los cultivares de desarrollo tardío) (Cuadro 3). Las muestras fueron secadas en un horno del laboratorio de Arvenses de la Facultad de Ciencias Agroalimentarias con el objetivo de preservar las características de la planta en el momento de realizar las mediciones. Se utilizó un estereoscopio trinocular marca Olympus $\AA^{\circledR}$ modeloSZ6145 para realizar mediciones a nivel de inflorescencia, principalmente a nivel de espiguillas. Se tomaron fotografías de las inflorescencias por 
medio del estereoscopio y una cámara fotográfica digital, para cada uno de los cultivares.

Cuadro 3. Características morfológicas de las inflorescencias de 13 cultivares de Brachiaria sp.

\begin{tabular}{lcccc}
\hline & & \multicolumn{3}{c}{ Inflorescencia } \\
\hline Especie-cultivar & Largo (cm) & Número & Largo racimos & Largo vaina (cm) \\
& & racimos & $(\mathrm{cm})$ & \\
B. decumbens-Basilisk & 45 & 3 & $7-11$ & $15,5-17,0$ \\
B. decumbens-Peludo & 23 & 3 & $9,5-10,0$ & 16 (peludo) \\
B. decumbens-Basilisk & 32 & 2 & 9,5 & $8,9-11,5$ \\
B. brizantha-Piatá & 48 & $3-5$ & $11-18$ & 18 \\
B. brizantha-MG-5 Victoria & 29 & 5 & $10,5-17,5$ & 19 \\
B. brizantha-Diamantes 1 & 45 & $2-3$ & 9 & 17 (peluda) \\
B. brizantha-Basilisk & 44 & 3 & 10 & $17,5-20,0$ \\
B. brizantha-Mulato & 34 & 3 & 9 & 16 \\
B. brizantha-Mulato 2 & 40 & 3 & $5,5-6,0$ & 16 (peluda) \\
B. brizantha-MG-5 Toledo & 43 & $4-5$ & $9,0-10,0$ & 19 (glabra) \\
B. brizantha-Marandú & 55 & $3-5$ & $13-16$ & $17,5-21,0$ (peluda) \\
B. brizantha-Toledo & 30 & 5 & $13,5-15,0$ & 17,5 \\
B. brizantha-Xaraes-Toledo & 36 & 3 & $19-23$ & \\
\hline
\end{tabular}




\section{RESULTADOS Y DISCUSIÓN}

\section{Información taxonómica de los cultivares de pasto Brachiaria}

En el cuadro 2 se muestra las características morfológicas de las hojas y los culmos de los 13 cultivares de pasto Brachiaria evaluados. Se debe tener presente que las características morfológicas mostradas en el presente estudio, se obtuvieron de plantas enteras cosechadas en estado vegetativo para las características de culmos y hojas y en estado de floración para recolectar las inflorescencias. Debido a las diferencias en los ciclos de crecimiento y floración de los diferentes cultivares, la cantidad de semanas fue diferente al momento de la cosecha entre los cultivares. En el caso de la recolección de culmos y hojas, la cosecha se realizó entre 20-35 días y las plantas no presentaban senescencia mientras que en floración se encontró más material senescente, principalmente hojas en los estratos inferiores.

El largo de las hojas fue entre 20 a $68 \mathrm{~cm}$ para $B$. decumbens cv. Basilisk y $B$. brizantha cv. MG-5 Victoria, respectivamente. Olivera et al (2006) indican que las hojas de $B$. decumbens tienen un largo entre 20 a $40 \mathrm{~cm}$ tal como se evidenció en el presente estudio, mientras que para $B$. brizantha, mencionan que el largo de las hojas puede llegar hasta $75 \mathrm{~cm}$ en algunos cultivares. Al igual que el largo de las hojas, el ancho se relaciona directamente con la capacidad fotosintética de las plantas; los cultivares $B$. decumbens cv. Basilisk y $B$. brizantha cv. Marandú y los dos de Toledo fueron los que mostraron un ancho superior a $2 \mathrm{~cm}$. Brachiaria decumbens tiene normalmente hojas con un ancho entre 1 a $2 \mathrm{~cm}$ que están cubiertas de tricomas (Olivera et al., 2006). Únicamente los cultivares Piatá, Diamantes y Basilisk de B. brizantha presentaron pubescencia en sus hojas, el resto de cultivares mostró hojas glabras, algunas de ellas son ásperas. Entre las accesiones de B. brizantha se puede encontrar diferentes hábitos de crecimiento (plantas erectas y rastreras) y cuyas hojas pueden ser pubescentes o glabras y con un ancho de 0,8 a 2,4 cm, rango dentro del que se encontraron los cultivares de B. brizantha del presente estudio (Olivera et al., 2006).

El largo de los entrenudos en los culmos varió desde $12 \mathrm{~cm}$ en $B$. decumbens cv. Basilisk hasta $34 \mathrm{~cm}$ en $B$. brizantha $\mathrm{cv}$. MG-5 Victoria. Los cultivares de $B$. brizantha cuyos culmos son erectos o sub erectos son escasamente ramificados, con 6 a 14 
entrenudos de 10 a $34 \mathrm{~cm}$ de longitud (Olivera et al., 2006), las mediciones obtenidas en el presente estudio se encuentran dentro de dicho rango.

El largo total del culmo tuvo un rango amplio, siendo el cultivar Peludo el de menor tamaño con 0,23 m (su culmo es peludo también) hasta 2,5 m para el cultivar Marandú de $B$. brizantha. Olivera et al. (2006) mencionan que el pasto $B$. decumbens puede tener una altura entre 30 a $100 \mathrm{~cm}$, con entrenudos de 18 a $28 \mathrm{~cm}$ de longitud; ambos rangos son similares a lo encontrado en el presente estudio para los cultivares Peludo y Basilisk. Los mismos autores indican que el pasto $B$. brizantha y sus accesiones, presentan macollas vigorosas cuyos culmos alcanzan hasta $2 \mathrm{~m}$ de altura, lo cual coincide con lo mostrado en el Cuadro 2 con excepción del cultivar Marandú que alcanzó $2,5 \mathrm{~m}$.

El cuadro 3 se muestra información a nivel de las inflorescencias de los cultivares de Brachiaria evaluados. El largo de la inflorescencia es variable con valores entre 23 hasta $55 \mathrm{~cm}$ para el pasto Peludo y Marandú, respectivamente. Olivera et al. (2006) mencionan que las inflorescencias de $B$. decumbens y $B$. brizantha son panículas compuestas de racimos y sus longitudes totales son entre 25 a $47 \mathrm{~cm}$ y entre 34 a 87 $\mathrm{cm}$, respectivamente.

La cantidad de racimos fue de 2-5 por inflorescencia; dicha característica parece estar relacionada con el tipo de cultivar, por lo tanto puede servir como medio de identificación entre los mismos. Así, los 3 cultivares de $B$. decumbens mostraron entre 2 a 3 racimos por inflorescencia y ambos cultivares de Mulato, mostraron valores consistentes en la cantidad de racimos pudiendo mostrar hasta 5 racimos (Olivera et al. 2006). Algunos cultivares de $B$. brizantha pueden llegar a tener hasta 17 racimos solitarios, unilaterales y rectos (Olivera et al., 2006), sin embargo, en este estudio la cantidad máxima de racimos por inflorescencia fue de 5 para los cultivares Marandú, Toledo y Piatá.

El largo de cada racimo fue entre 5,5cm para el pasto Mulato 2 y $23 \mathrm{~cm}$ para el cultivar Xaraes-Toledo. Los cultivares de $B$. decumbens mostraron un largo en los racimos mayor al reportado por Olivera et al. (2006) con 4 a $10 \mathrm{~cm}$ de largo, mientras que los cultivares de $B$. brizantha se encontraron, en su mayoría, dentro del rango de 8 a $22 \mathrm{~cm}$ reportado por los mismos autores. El largo de la vaina en las inflorescencias de pasto 
Brachiaria fue generalmente menor en los cultivares con menor número y largo de racimos; así el $B$. decumbens cv. Basilisk mostró una vaina cercana a $9 \mathrm{~cm}$ mientras que en el $B$. brizantha $\mathrm{cv}$. Toledo su vaina fue de $21 \mathrm{~cm}$ de largo.

El Cuadro 4 incluye una explicación detallada sobre las características encontradas en el raquis y las espiguillas de los cultivares de Brachiaria evaluados. Como complemento a la información descrita en dicho cuadro, se puede utilizar las Figuras de la 2 a la 6 , cuyas fotografías pretenden ser una herramienta de identificación en campo para los cultivares al llegar al estado de floración.

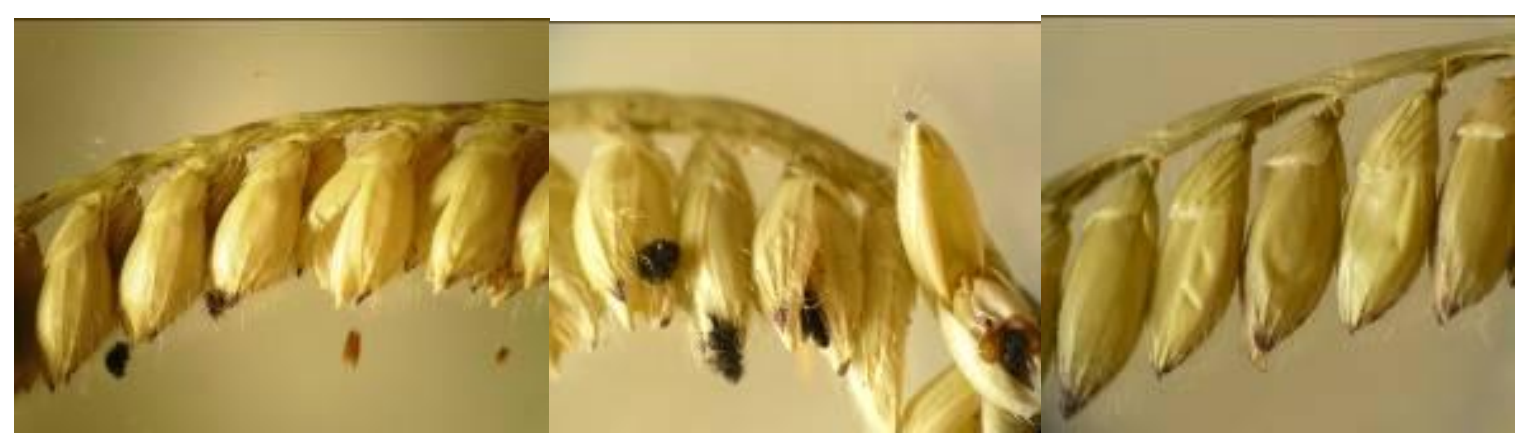

Figura 2. Inflorescencias de cultivares de Brachiaria decumbens (P1, P2 y P3) tomadas con estereoscopio. 
Cuadro 4. Características morfológicas de las inflorescencias de 13 cultivares de pasto Brachiaria.

\begin{tabular}{|c|c|c|}
\hline $\begin{array}{l}\text { Especie-cultivar } \\
\text { (parcela) }\end{array}$ & Raquis & Espiguillas \\
\hline $\begin{array}{l}\text { B. decumbens- } \\
\text { Basilisk (P1) }\end{array}$ & $\begin{array}{l}\text { Presenta leve torsión y } \\
\text { pubescente en ambos } \\
\text { bordes }\end{array}$ & $\begin{array}{l}5 \mathrm{~mm} \text { largo, pubescentes de la mitad } \\
\text { hacia el ápice, glumas con más de } 8 \\
\text { nervios, } 1 \text { flor hermafrodita y una } \\
\text { masculina estéril }\end{array}$ \\
\hline $\begin{array}{l}\text { B. decumbens-Peludo } \\
\text { y Basilisk (P2 y P3) }\end{array}$ & Velludo en ambos bordes & $\begin{array}{l}5 \mathrm{~mm} \text { largo, pubescentes de la mitad } \\
\text { hacia el extremo, flores hermafroditas }\end{array}$ \\
\hline $\begin{array}{l}\text { B. brizantha-Piatá } \\
\text { (P4) }\end{array}$ & $\begin{array}{l}\text { Pelos en ambos márgenes } \\
\text { de forma irregular }\end{array}$ & $\begin{array}{l}7 \mathrm{~mm} \text { largo, } 1 \text { gluma pequeña con } 10 \text { o } \\
\text { más nervios, glabras y el ápice de la } \\
\text { espiguilla con una coloración negra, flores } \\
\text { hermafroditas }\end{array}$ \\
\hline $\begin{array}{l}\text { B. brizantha-MG-5 } \\
\text { Victoria (P5) }\end{array}$ & $\begin{array}{l}\text { Pocos pelos en ambos } \\
\text { márgenes }\end{array}$ & $\begin{array}{l}7 \mathrm{~mm} \text { largo, pelos de la mitad hacia el } \\
\text { ápice y éste de color oscuro, flores } \\
\text { hermafroditas y estériles }\end{array}$ \\
\hline $\begin{array}{l}\text { B. brizantha- } \\
\text { Diamantes } 1 \text { (P6) }\end{array}$ & $\begin{array}{l}\text { Pelos largos en ambos } \\
\text { bordes }\end{array}$ & $\begin{array}{l}7 \mathrm{~mm} \text { largo con } 1 \text { gluma pequeña, pelos } \\
\text { de la mitad hacia el ápice de las } \\
\text { espiguillas, ápice oscuro, flores } \\
\text { hermafroditas y estériles }\end{array}$ \\
\hline $\begin{array}{l}\text { B. brizantha-Basilisk } \\
\text { (P7) }\end{array}$ & Pelos en ambos bordes & $\begin{array}{l}8 \mathrm{~mm} \text { largo, pelos de la mitad hacia el } \\
\text { ápice, flores hermafroditas y masculinas }\end{array}$ \\
\hline $\begin{array}{l}\text { B. brizantha-Mulato } \\
\text { (P8) }\end{array}$ & Pelos en ambos márgenes & $\begin{array}{l}5 \mathrm{~mm} \text { largo, glabras, ápice color negro y } \\
\text { con muy pocos pelos, flores hermafroditas } \\
\text { y masculinas }\end{array}$ \\
\hline $\begin{array}{l}\text { B. brizantha-Mulato } 2 \\
\text { (P9) }\end{array}$ & $\begin{array}{l}\text { Pelos largos a ambos } \\
\text { lados }\end{array}$ & $\begin{array}{l}5 \mathrm{~mm} \text { largo, pocos pelos solo en el ápice, } \\
\text { espiguillas de color verde, flores } \\
\text { hermafroditas y estériles }\end{array}$ \\
\hline $\begin{array}{l}\text { B. brizantha-MG-5 } \\
\text { Toledo (P10) }\end{array}$ & $\begin{array}{l}\text { Pocos pelos (1-2 en medio } \\
\text { de las espiguillas) }\end{array}$ & Flores hermafroditas y algunas estériles \\
\hline $\begin{array}{l}\text { B. brizantha-Marandú } \\
(\mathrm{P} 11)\end{array}$ & $\begin{array}{l}\text { Muy velludo en ambos } \\
\text { márgenes }\end{array}$ & $\begin{array}{l}7 \mathrm{~mm} \text { largo con pocos pelos en el ápice, } \\
\text { flores hermafroditas algunas estériles }\end{array}$ \\
\hline $\begin{array}{l}\text { B. brizantha-Toledo } \\
\text { (P12) }\end{array}$ & $\begin{array}{l}\text { Pocos pelos en sus } \\
\text { márgenes }\end{array}$ & $\begin{array}{l}7 \mathrm{~mm} \text { largo, pelos terminales en el ápice, } \\
\text { flores hermafroditas algunas estériles }\end{array}$ \\
\hline $\begin{array}{l}\text { B. brizantha-Xaraes- } \\
\text { Toledo (P13) }\end{array}$ & $\begin{array}{l}\text { Muchos pelos en sus } \\
\text { márgenes }\end{array}$ & $\begin{array}{l}5 \mathrm{~mm} \text { largo, ápice de color oscuro y con } \\
\text { pelos, espiguillas hermafroditas y estériles }\end{array}$ \\
\hline
\end{tabular}


Los tres cultivares de $B$. decumbens mostraron pubescencia en ambos bordes del raquis del cual cuelgan las espiguillas, las cuales se mencionan como oblongo-elípticas de 3 a $4 \mathrm{~mm}$ de largo (Olivera et al., 2006), mientras en el presente estudio mostraron un largo de $5 \mathrm{~mm}$ (Figura 2). Los tres cultivares Piatá, MG-5 Victoria y Basilisk de $B$. brizantha tienen raquis pubescentes, Piatá tiene espiguillas glabras y una coloración negra en el ápice, mientras que en los otros dos cultivares sus espiguillas son pubescentes (Figura 3).

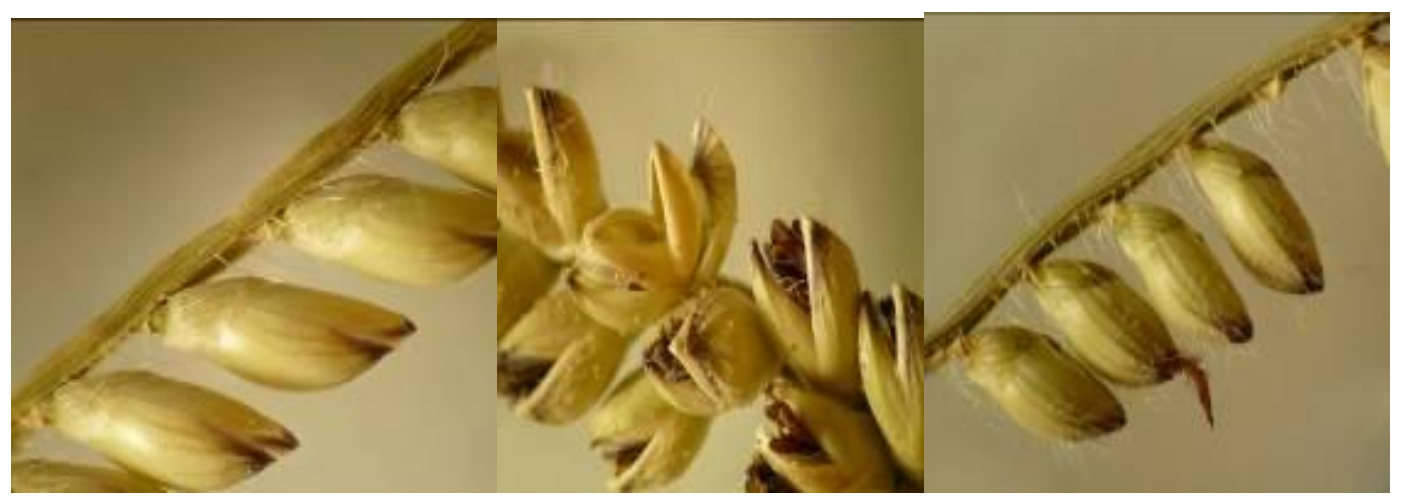

Figura 3. Inflorescencias de cultivares de Brachiaria brizantha (P4-Piatá, P5-MG-5 Victoria y P7-Basilisk) tomadas con estereoscopio.

Como se mencionó antes, algunos de los cultivares de Brachiaria presentan características particulares que pueden facilitar su identificación a nivel de campo; tal como sucede con los cultivares de $B$. brizantha Diamantes 1 y Marandú, donde el segundo presenta un mayor grado de pubescencia en el raquis que sostiene las espiguillas, a pesar de que ambos representan el mismo cultivar con nombres comunes diferentes de acuerdo al origen de la semilla, dígase para Costa Rica y Suramérica, respectivamente (Figura 4). 


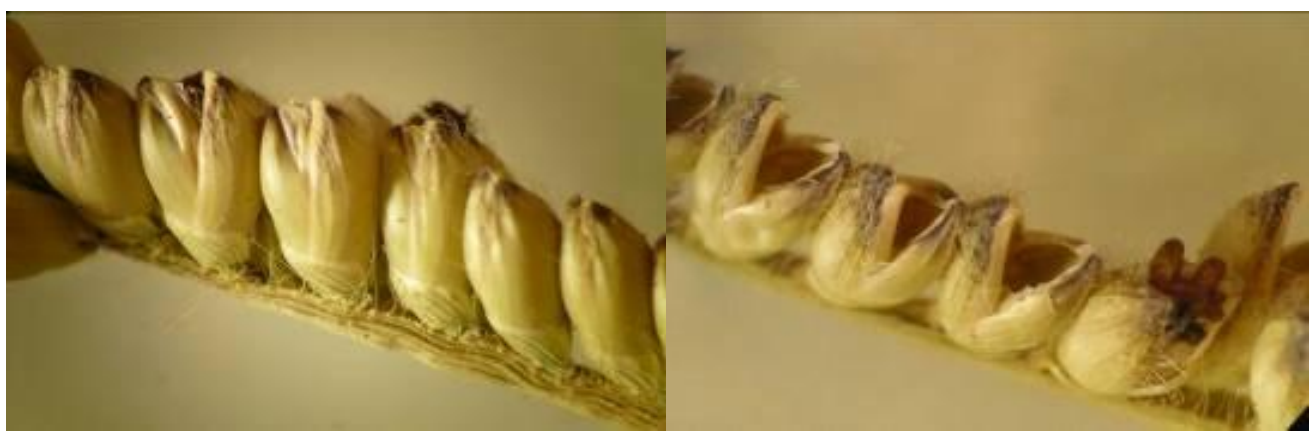

Figura 4. Inflorescencias de cultivares de Brachiaria brizantha (P6-Diamantes 1 y P11Marandú) tomadas con estereoscopio.

El cultivar de $B$. brizantha Mulato 1 tiene su ápice de color negro, característica que no está presente en el Mulato 2 (Figura 5); el primero fue el primer híbrido apomíctico comercial obtenido por el CIAT y se ha destacado su adaptación a una amplia gama de localidades (Argel 2006).

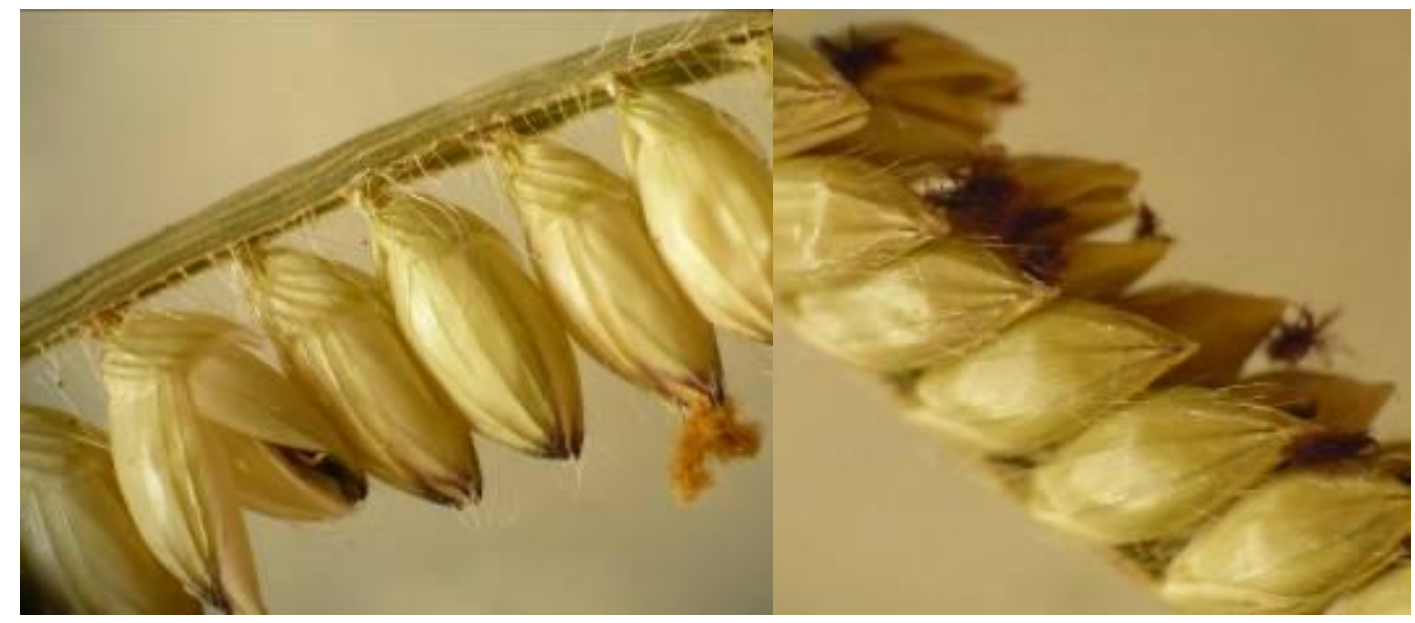

Figura 5. Inflorescencias de dos cultivares mulato de Brachiaria (P8-Mulato 1 y P9Mulato 2) tomadas con estereoscopio.

Los tres cultivares de pasto Toledo ( $B$. brizantha) evaluados mostraron diferencias en pubescencia en el raquis de la inflorescencia, siendo menor en MG-5 Toledo, y Toledo con respecto a Xaraes-Toledo (Figura 6). La utilización de un estereoscopio facilita el reconocimiento detallado de las características tal como se hizo en el presente estudio; sin embargo por medio de un lente ocular (lupa) en conjunto con las características 
taxonómicas, se puede facilitar la identificación de los cultivares de Brachiaria en campo.

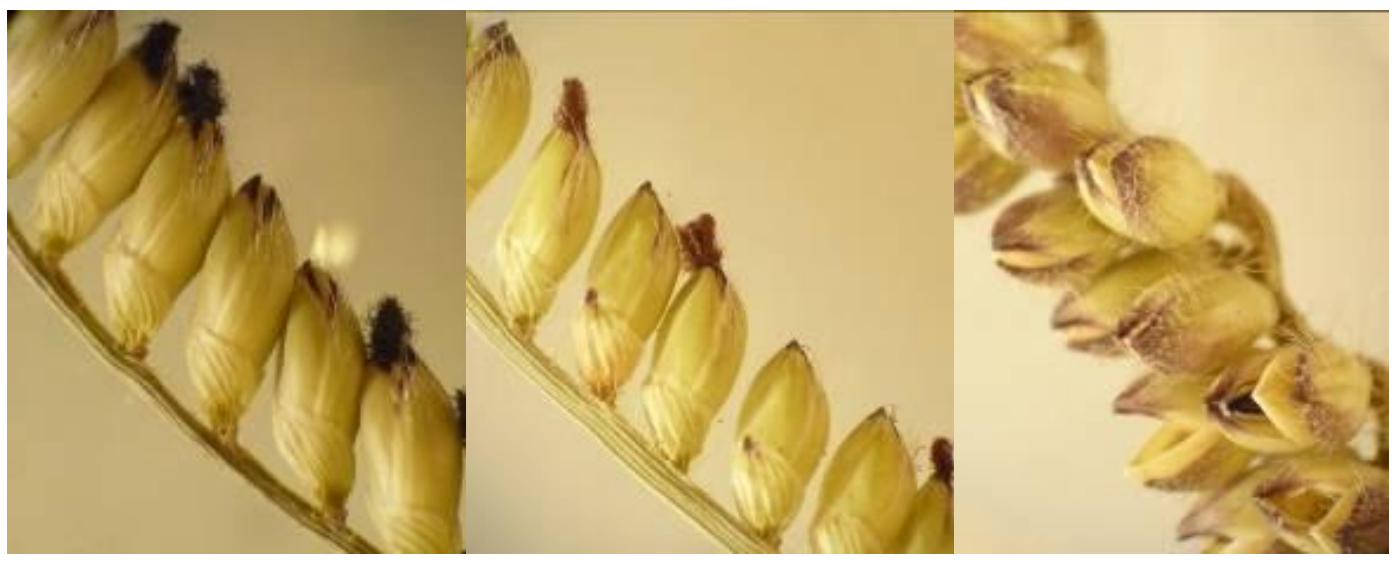

Figura 6. Inflorescencias de tres cultivares de Brachiaria brizantha (P10-MG-5 Toledo, P12-Toledo y P13-Xaraes-Toledo) tomadas con estereoscopio.

La información mostrada en este estudio sirve como herramienta taxonómica para evaluar las especies del género Brachiaria. A pesar de que mucha de esta información podría no ser sencilla de medir en campo, las características taxonómicas pueden utilizarse para diagnósticos por descarte, pasando primero por las características macroscópicas propias ,a nivel de culmo y hoja; y utilizando las características de las inflorescencias si se requiere confirmar la identificación de un cultivar específico.

La selección en pastos se basa primordialmente en criterios agronómicos y, salvo raras excepciones, se considera la parte taxonómica y/o morfológica, sin embargo éstas últimas se van depurando al mismo tiempo durante el proceso. La presente investigación evidencia como, cultivares pertenecientes a la misma especie con diferente número de accesión, muestran características taxonómicas diferentes.

\section{CONSIDERACIONES FINALES}

El género Brachiaria representan una de las principales fuentes de forraje para rumiantes en condiciones tropicales, sin embargo existe poca información disponible sobre las características taxonómicas de los cultivares disponibles en el mercado. La 
mayoría de información se limita a las especies más comúnmente utilizadas, mientras que para los cultivares desarrollados con sus diferentes accesiones liberadas al mercado en los últimos años, se tiene mayor facilidad de acceso a información agronómica.

Existen características morfológicas de los pastos del género Brachiaria que se han utilizado como criterio de selección y, éstas características a su vez, han modificado otros componentes estructurales de la planta con respecto a sus antecesores, los cuales pudieron ser clones, como la mayoría de accesiones, o cruces entre especies fértiles.

La presente investigación brinda una herramienta de identificación de los cultivares de Brachiaria que puede ser utilizada por productores para identificación de cultivares en sus terrenos. Asimismo, técnicos de instituciones públicas y privadas involucrados con la comercialización de semillas, podrían utilizarlo como complemento en los procesos de control de calidad de las semillas que ingresan a Costa Rica.

\section{AGRADECIMIENTOS}

Los autores expresan su agradecimiento al Ing. Orlando Carrillo de la Oficina Nacional de Semillas, al M.Sc. Ramiro Alizaga, Director del Centro de Investigaciones en Granos y Semillas y a la Estación Experimental Fabio Baudrit por la colaboración brindada para la realización de la investigación.

\section{LITERATURA CITADA}

Argel, P.J. 2006. Contribución de los forrajes mejorados a la productividad ganadera en sistemas de doble propósito. Archivos Latinoamericanos de Producción Animal 14(2):65-75.

Carrillo, O. 2012. Verificación de Estándares de Calidad de semilla de especies forrajeras, pp. 41-43. In: Memoria Anual 2012. Oficina Nacional de Semillas. 
CENTRO INTERNACIONAL DE AGRICULTURA TROPICAL (CIAT). 2010. Ganado, Cambio Climático y Brachiaria. Hoja Informativa $\mathrm{n}^{\circ} 12$. In:http://www.ciat.cgiar.org

Cook, B.G., Pengelly, B.C., Brown, S.D., Donnelly, J.L., Eagles, D.A., Franco, M.A., Hanson, J., Mullen, B.F., Partridge, I.J., Peters, M., Schultze-Kraft, R. 2005. Tropical Forages: an interactive selection tool, [CD-ROM], CSIRO, DPI\&F (QId), CIAT and ILRI, Brisbane, Australia.

ESTACIÓN EXPERIMENTAL AGRÍCOLA FABIO BAUDRIT MORENO (EEFBM). 2014. Ubicación geográfica. In: http://www.eefb.ucr.ac.cr/esp_ubicacion.shtml

Hernández, M., Herrera, F. 2005. Respuesta de los pastos Panicum maximum, Brachiaria brizantha y $B$. decumbens a herbicidas posemergentes con acción graminicida. Revista de Agricultura Tropical 35:15-25.

Hickey, M., Clive, K. 2000.The Cambridge illustrated glossary of botanical terms. Cambridge University Press.20 p.

INSTITUTO METEOROLÓGICO NACIONAL (IMN). 2012. Datos climáticos para el cantón de Alajuela de 1999 al 2012. Estación 169, Aeropuerto Juan Santamaría. In:http://www.imn.ac.cr/IMN/MainAdmin.aspx?_EVENTTARGET=ClimaCiudad\& CIUDAD $=8$

Janzen, D.H. 1991. Historia natural de Costa Rica. 1ª ed. Editorial de la UCR. San José, Costa Rica. 822 p.

Olivera, Y., Machado, R., Del Pozo, P.P. 2006. Características botánicas y agronómicas de especies forrajeras importantes del género Brachiaria. Pastos y Forrajes 29(1):1-23.

Retana, J.A., Rosales, R. 2000. Efecto de la variabilidad climática en la Región Chorotega sobre la producción bovina de carne en Costa Rica. Tópicos Meteorológicos y Oceanográficos 7(1):1-20.

Villalobos, L., Arce, J., Wingching, R. 2013. Producción de biomasa y costos de producción de pastos estrella africana (Cynodon nlemfuensis), kikuyo (Kikuyoucloa clandestinum) y ryegrass perenne (Lolium perenne) en lecherías de Costa Rica. Agronomía Costarricense 37(2):91-103.

Villalobos, L., Arce, J., Wingching, R. 2014. Costos de producción de ensilajes elaborados con pastos de piso, de corte y anuales en lecherías de Costa Rica. En prensa. 
Wingching, R., Salazar, L., Flores, L., Rojas, A. 2008. Reconocimiento de nemátodos en pastos tropicales en las comunidades de Sucre y San Vicente, Cantón de San Carlos. Agronomía Costarricense 32(2):129-136.

Zuleta, C., Kelemu, S., Cardozo, O. 2002. Identificación de fuentes de resistencia a Xanthomonas campestris en Brachiaria spp. Manejo Integrado de Plagas y Agroecología (Costa Rica) 64:41-47.

\section{GLOSARIO *}

Accesión: número asignado a un eco tipo (cultivar) de una especie cuando su germoplasma e información de procedencia es almacenado y mantenido para el futuro a través de centros de recursos genéticos tales como CIAT e ILRI.

Ápice: extremo de un órgano.

Culmos: término utilizado para los tallos de las gramíneas (pastos).

Decumbente: forma de crecimiento de los tallos tendidos sobre el suelo con el ápice erguido.

Entrenudos: parte del tallo comprendida entre dos nudos.

Espiguillas: inflorescencia de las gramíneas.

Glabra(o): desprovisto de pelo.

Inflorescencias: conjunto de flores que nacen de un sistema ramificado.

Macollas: conjunto de ramificaciones o vástagos nacidos en la base de un mismo pie.

Nudos: engrosamiento del tallo donde se originan yemas, hojas, ramas, etcétera.

Pubescente: órgano cubierto de pelos finos y suaves.

Racimos: inflorescencia con un eje principal de crecimiento indefinido, del que brotan flores desde la base hasta el ápice.

Raquis: eje del que nacen los foliolos (segmentos) de una hoja compuesta o las flores de una inflorescencia.

Senescencia: estado de inactividad fisiológica en plantas luego alcanzar la madurez.

Tricomas: formación epidérmica que resalta en la superficie de los órganos vegetales.

Vainas: parte basal de algunas hojas (por ejemplo gramíneas) que envuelve total o parcialmente el tallo.

* Definiciones tomadas de Hickey y Clive (2000). 